\title{
Type I collagen deposition via osteoinduction ameliorates YAP/TAZ activity in 3D floating culture clumps of mesenchymal stem cell/extracellular matrix complexes
}

Nao Komatsu ${ }^{\dagger}$, Mikihito Kajiya ${ }^{*+}$ (D), Souta Motoike, Manabu Takewaki, Susumu Horikoshi, Tomoyuki Iwata, Kazuhisa Ouhara, Katsuhiro Takeda, Shinji Matsuda, Tsuyoshi Fujita and Hidemi Kurihara

\begin{abstract}
Background: Three-dimensional (3D) floating culture clumps of mesenchymal stem cell (MSC)/extracellular matrix (ECM) complexes (C-MSCs) consist of cells and self-produced ECM. Previous studies have demonstrated that C-MSCs can be transplanted into bony lesions without an artificial scaffold to induce bone regeneration. Moreover, osteoinductive medium (OIM)-treated C-MSCs (OIM-C-MSCs) have shown rapid and increased new bone formation in vivo. To apply OIM-C-MSCs for novel bone regenerative cell therapy, their cellular properties at the molecular level must be elucidated. The transcriptional co-activators yes-associated protein/transcriptional co-activator with PDZ-binding motif (YAP/TAZ) have been recognized as key players in the mechanotransduction cascade, controlling cell lineage commitment in MSCs. It is plausible that 3D C-MSCs/OIM-C-MSCs cultured in floating conditions could provide distinct microenvironments compared to conventional 2D culture systems and thereby induce unique mechanotransduction cascades. Therefore, this study investigated the YAP/TAZ activity in 3D-cultured C-MSCs/ OIM-C-MSCs in floating conditions.
\end{abstract}

Methods: Human bone marrow-derived MSCs were cultured in growth medium supplemented with ascorbic acid. To obtain C-MSCs, confluent cells that had formed on the cellular sheet were scratched using a micropipette tip and were then torn off. The sheet was rolled to make round clumps of cells. Then, YAP/TAZ activity, filamentous actin (F-actin) integrity, collagen type I (COL1) production, and the differentiation potency in 3D floating culture C-MSCS/OIM-C-MSCs were analyzed.

Results: C-MSCs cultured in floating conditions lost their actin cytoskeleton to downregulate YAP/TAZ activity, which directed cells to undergo adipogenesis/chondrogenesis. OIM treatment induced abundant COL1 deposition, which facilitated Int $\beta 1$-dependent actin fiber formation and YAP/TAZ activity to elevate the expression levels of osteogenic master transcriptional factor runt-related transcription factor 2 (RUNX2) mRNA in C-MSCs. Importantly, elevation of YAP/TAZ activity via OIM was associated with COL1 deposition and F-actin integrity, suggesting a positive feedback loop in OIM-C-MSCS.

Conclusion: These findings suggest that OIM-C-MSCS, which form a unique microenvironment that maintains high YAP/TAZ activity, can serve as better candidates for bone regenerative cell therapy than C-MSCs.

Keywords: Mechanotransduction, 3D culture, YAP/TAZ, F-actin, C-MSCS

\footnotetext{
*Correspondence: mkajiya@hiroshima-u.ac.jp

${ }^{\dagger}$ Nao Komatsu and Mikihito Kajiya contributed equally to this work.

Department of Periodontal Medicine, Applied Life Sciences, Institute of

Biomedical \& Health Sciences, Graduate School of Biomedical \& Health

Sciences, Hiroshima University, Minami-ku, Kasumi 1-2-3, Hiroshima,

Hiroshima 734-8553, Japan
}

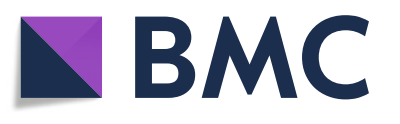

(c) The Author(s). 2018 Open Access This article is distributed under the terms of the Creative Commons Attribution 4.0 International License (http://creativecommons.org/licenses/by/4.0/), which permits unrestricted use, distribution, and reproduction in any medium, provided you give appropriate credit to the original author(s) and the source, provide a link to the Creative Commons license, and indicate if changes were made. The Creative Commons Public Domain Dedication waiver (http://creativecommons.org/publicdomain/zero/1.0/) applies to the data made available in this article, unless otherwise stated. 


\section{Background}

Mesenchymal stem cells (MSCs), which possess selfrenewing properties, multipotencey, and immunomoduratory potential, are currently the most extensively studied cells for tissue regenerative cell therapy, mainly due to their relative ease of isolation compared to other multipotent cells, such as embryonic stem cells and induced pluripotent stem cells [1]. For the tissue regenerative cell therapy, one of the common approaches is to seed MSCs on scaffold or microcarrier. However, despite recent advances, usage of the artificial scaffolds still harbors several limitations, including biodegradability and host unfavorable inflammatory response [2]. Indeed, our previous study demonstrated that MSC transplantation with osteointegrative biomaterial, $\beta$-tricalcium phosphate, induced alveolar bone regeneration in dog class III furcation defect model, though ankylosis, which is an abnormal adhesion of bone and periodontal ligament, was observed [3]. Accordingly, to solve this problem, we recently established clumps of MSC/extracellular matrix (ECM) complexes (C-MSCs), which consist of cells and self-produced ECM [4-7]. Three-dimensional (3D)-cultured C-MSCs can be grafted into bony lesions without any artificial scaffolds to induce bone regeneration. In addition, osteoinductive medium (OIM)-treated C-MSCs (OIM-C-MSCs) show rapid and increased new bone formation $[4,6]$. These previous findings have suggested that OIM-C-MSCs may provide a promising bone regenerative therapy that utilizes cells while avoiding problems associated with the biocompatibility/biodegradability of artificial scaffolds. To develop such a novel cell therapy based on scientific evidence, it is necessary to distinguish the biological capacities of C-MSCs and OIM-C-MSCs at the molecular level.

Compared to traditional cell monolayers cultured on two-dimensional (2D) plastic or glass plates, 3D cultures of C-MSCs could provide markedly different and unique microenvironments for cells. For instance, 2D collagen-coated plastic plates provide a high-stiffness substrate, a continuous layer of ECM, and sufficient space for cells to spread [8,9]. Conversely, 3D culture systems using various types of gels exhibit decreased stiffness, discrete matrix fibrils, and limited space, restricting cell shapes to a rounded form [8,9]. Importantly, using mechanotransduction systems, such as those including integrins and cytoskeletal tension, cells can translate physical and mechanical cues into biochemical signals to regulate cell growth and differentiation. Indeed, cell density, cell shape, and matrix elasticity have been shown to direct MSC lineage commitment via cytoskeletal mechanics [10-12]. Accordingly, it is conceivable that 3D C-MSCs cultured in floating conditions could provide distinct microenvironments compared to conventional $2 \mathrm{D}$ culture systems, thereby resulting in the induction of a unique mechanotransduction pathway that regulates cell lineage specification and function.

The transcriptional co-activators yes-associated protein (YAP) and transcriptional co-activator with PDZ-binding motif (TAZ), originally identified as downstream targets of the Hippo signaling pathway [13-15], have recently been recognized as key factors in the mechanotransduction cascade [16-18]. Cellular microenvironments that tend to produce high intracellular resistive forces via actin fibers, including an extensive adhesion area, low cell density, stiff ECM, or fluid shear stress, facilitate YAP/TAZ localization to the nucleus to activate their transcriptional activities. In contrast, a narrow adhesion area, high cell density, soft ECM, and disturbed fluid flow produce a low cytoskeletal contractile force by disrupting the integrity of actin fibers, which causes YAP/TAZ cytoplasmic retention and proteasomal degradation $[15,19,20]$. Intriguingly, DuPont et al. reported that MSCs, by sensing the mechanical cues that facilitate the integrity of actin (e.g., stiff ECM), were induced to form osteoblasts via high YAP/TAZ activity, whereas cells cultured on soft ECM or treated with an acto-myosin inhibitor showed low YAP/TAZ activity, consistent with adipogenesis [21].

Based on these accumulating lines of evidence, we hypothesized that the microenvironment surrounding $3 \mathrm{D}$ floating cultured C-MSCs, which should be different from traditional 2D culture, may cause unique mechanotransduction through cytoskeletal mechanics and YAP/ TAZ activity, which, in turn, affects the direction of cells' lineage commitment. Accordingly, the present study investigated (1) filamentous actin (F-actin) fiber integrity and YAP/TAZ activity in 3D floating culture C-MSCs and (2) the effects of OIM on the mechanotransduction cascade that underlies the molecular mechanism of osteogenic induction in C-MSCs.

\section{Materials and methods C-MSC preparation and culture}

Human bone marrow MSCs were purchased from Lonza (Lonza, Basel, Switzerland) or were provided by RIKEN BioResource Center (Ibaragi, Tsukuba, Japan). The cells were cultured in DMEM (Sigma, Steinheim, Germany) supplemented with $10 \%$ fetal bovine serum (FBS) (HyClone, Logan, UT), $100 \mathrm{U} / \mathrm{mL}$ penicillin (Sigma), and $100 \mu \mathrm{g} / \mathrm{mL}$ streptomycin (Sigma). Then, C-MSCs were generated as described previously [5]. Briefly, MSCs were seeded at a density of $2.0 \times 10^{5}$ cells/well in 24-well plates (Corning, Corning, NY) and cultured with high-glucose DMEM (Sigma) supplemented with $10 \%$ fetal FBS, $100 \mathrm{U} / \mathrm{mL}$ penicillin, and $100 \mu \mathrm{g} / \mathrm{mL}$ streptomycin (growth medium $(\mathrm{GM})$ ) in the presence of $50 \mu \mathrm{g} / \mathrm{mL}$ L-ascorbic acid (Sigma) for 4 days. To obtain 
C-MSCs, we took confluent cells that had formed a cellular sheet, consisting of self-produced ECM, and detached them from the bottom of the plate by scratching the edge with a micropipette tip. The floating MSC/ECM complexes were transferred to a 24-well ultra-low-binding plate (Corning) and cultured with GM or OIM (GM supplemented with $100 \mathrm{nM}$ dexamethasone (Sigma), $50 \mu \mathrm{g} / \mathrm{mL} \mathrm{L}$-ascorbic acid, and $10 \mathrm{mM} \beta$-glycerophosphate (Sigma)) in the presence or absence of Y27632 (50 $\mathrm{MM}$, Chemdea, Ridgewood, NJ) or blebbistatin ( $50 \mu \mathrm{M}$, Cayman Chemical, Ann Arbor, MI) for 5 days. After 1 day of incubation, the cellular sheets rolled up, producing round clumps of cells, and 0.8-1.2-mm-diameter C-MSCs were obtained (Fig. 1a). All experiments were repeated at least three times independently.

\section{Transfection}

siRNAs targeting YAP, TAZ, or negative control siRNA [21] were chemically synthesized and provided by FASMAC (FASMAC, Kanagawa, Japan). The sequences of these siRNAs are listed in Additional file 1: Table S1. Validated integrin $\beta 1$ (Int $\beta 1)$ siRNA was obtained from Invitrogen (\#HSS105561; Invitrogen, Carlsbad, CA). Two days before the production of C-MSCs (before scratching), siRNAs were transfected into cells using Lipofectamine RNAi-MAX (Invitrogen) according to the manufacturer's instructions. Then, the cell sheets were detached and transferred to a 24-well ultra-low-binding plate to generate C-MSCs as described above. The knockdown effects of each siRNA were assessed (Additional file 2:

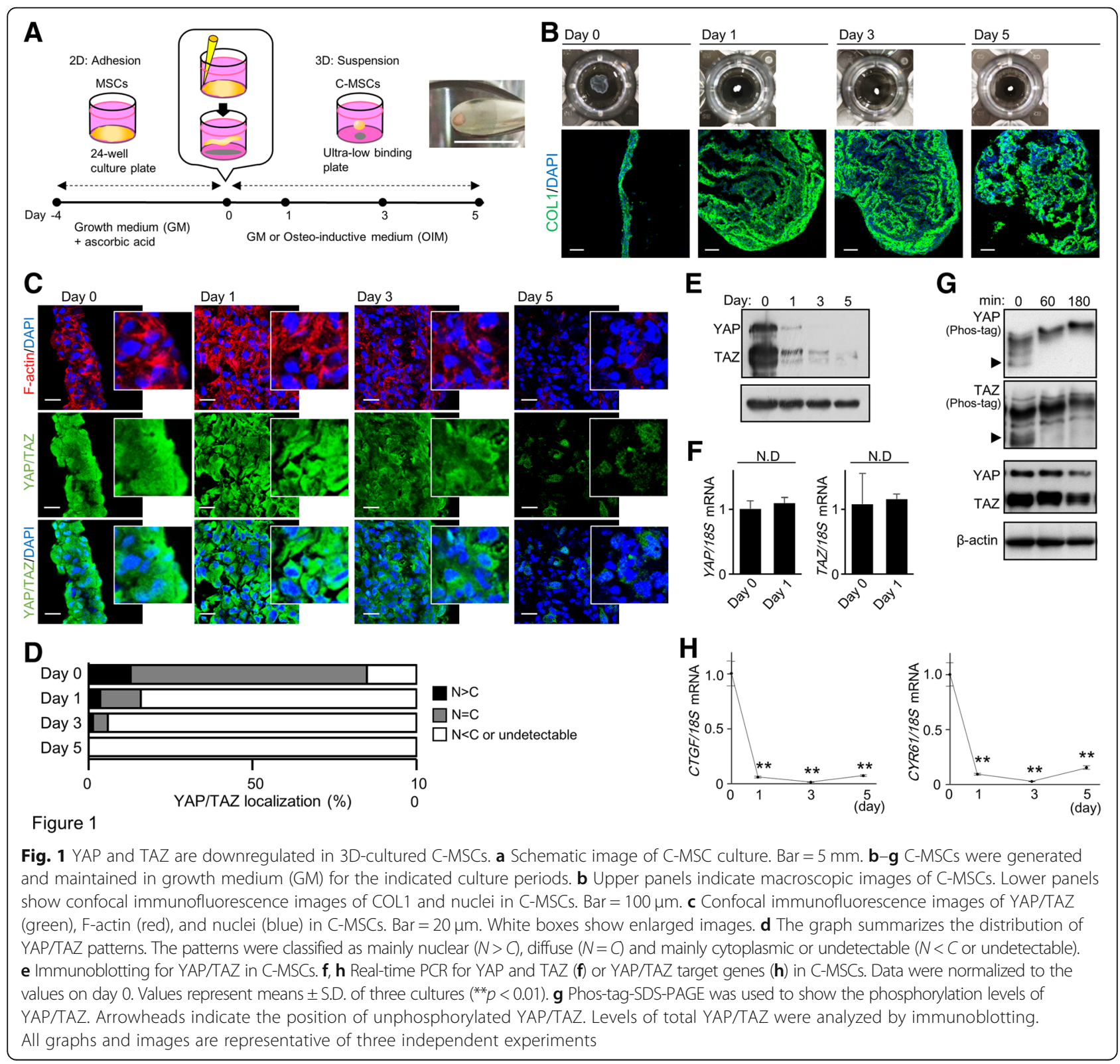


Figure S1A-S1C). All experiments were repeated at least three times independently.

The expression plasmid for HA-TAZS89A was obtained from Addgene (Addgene plasmid 32840; Addgene, Cambridge, MA). An HA expression plasmid was employed as a control (\#635688; Clontech, Palo Alto, CA). DNA transfections were performed with ECM 830 (BTX, Holliston, MA). Briefly, after detaching the MSC/ECM complexes from the culture plate, the cell sheet was suspended in $100 \mu \mathrm{L}$ of Opti-MEM (Invitrogen) with $4 \mu \mathrm{g}$ of each DNA plasmid and placed into 4-mm-gap cuvettes. Transfections were conducted at $300 \mathrm{~V}$ with a $1 \mathrm{~ms}$ pulse length and 10 pulses over $5 \mathrm{~s}$. After the pulses, the cell sheets were transferred to a 24-well ultra-low-binding plate to generate C-MSCs, as described above. The effects of each plasmid transfection were assessed (Additional file 2: Figure S1D and S1E). All experiments were repeated at least three times independently.

\section{Induction of C-MSCs}

For osteogenic, adipogenic, or chondrogenic induction, the cell sheets were detached from the culture plates and maintained in OIM, adipogenic induction medium (AIM; high-glucose DMEM supplemented with $10 \%$ FBS, $0.5 \mathrm{mM}$ isobutylmethylxanthine (Nacalai Tesque, Kyoto, Japan), $0.1 \mathrm{mM}$ indomethacin (Sigma), $1 \mu \mathrm{M}$ dexamethasone (Sigma), and $10 \mu \mathrm{g} / \mathrm{mL}$ insulin (Sigma)), chondrogenic induction medium (CIM; high-glucose DMEM supplemented with ITS $^{\text {Tix }}+$ Premix (Corning), 1 $\mathrm{mM}$ sodium pyruvate (Sigma), $0.35 \mathrm{mM}$ proline (Sigma), $10 \mathrm{ng} / \mathrm{mL}$ transforming growth factor- $\beta 3$ (Aviscera Bioscience, Santa Clara, CA), $100 \mathrm{nM}$ dexamethasone, and $0.17 \mathrm{mML}$-ascorbic acid), or dual induction medium (dual; equal volumes of OIM and AIM) [22] for 5 days. All experiments were repeated at least three times independently.

\section{Immunofluorescence analysis}

C-MSCs were fixed with $4 \%$ paraformaldehyde in PBS. The fixed samples were embedded in Tissue-Tek OCT compound (Sakura, Torrance, CA), and $20-\mu \mathrm{m}$-thick sections were cut using a cryostat. The sections were washed with PBS and then treated with 5\% BSA and 0.1\% TWEEN 20 in PBS to block nonspecific staining. These sections were incubated with a rabbit anti-human YAP/ TAZ IgG antibody (D24E4; 1:1000; Cell Signaling, Beverly, MA) or rabbit anti-human type I collagen polyclonal IgG (1:200; Novus Biologicals, Littleton, CO) at $4{ }^{\circ} \mathrm{C}$ overnight. After being washed 3 times with PBS, samples were incubated for $2 \mathrm{~h}$ with an Alexa Fluor $488^{\circ}$ goat anti-rabbit IgG antibody (1:100; Invitrogen) and Alexa Fluor 594 phalloidin (1:50; Invitrogen) at room temperature. Then, the nuclei were counterstained with DAPI $(5 \mu \mathrm{g} / \mathrm{mL}$; Invitrogen). After washing the samples with PBS, we detected the fluorescence signals using a Zeiss LSM 5 PASCAL laser scanning confocal microscope (Carl Zeiss Microimaging, Inc., Oberkochen, Germany). To assess YAP/TAZ distribution patterns, we quantified cells in three random fields of view ( $n=150 \sim 200)$ as previously described [23].

\section{Immunoblotting}

The C-MSCs were lysed in buffer containing $25 \mathrm{mM}$ Tris-HCl (pH 7.4), $150 \mathrm{mM} \mathrm{NaCl}, 5 \mathrm{mM}$ EDTA (pH 8.0), $0.1 \%$ SDS, $1 \%$ NP-40, $10 \%$ glycerol, and $1 \%(v / v)$ Triton $\mathrm{X}-100$ [24]. The cell lysates were subjected to ultrasonic treatment for $8 \mathrm{~s}$ on ice. Proteins in the lysates were subjected to SDS-PAGE and transferred to a nitrocellulose membrane (Bio-Rad Laboratories, Hercules, CA). The membranes were blocked for $1 \mathrm{~h}$ with $5 \%$ skim milk and then immunoblotted using rabbit anti-YAP/TAZ IgG (D24E4; 1:1000; Cell Signaling), rabbit anti-YAP IgG (D8H1X; 1:1000; Cell Signaling), rabbit anti-TAZ IgG (V386; 1:1000; Cell Signaling), or mouse anti- $\beta$-actin monoclonal antibodies (AC-15; 1:10000; Sigma). The membranes were developed using ECL Plus Western blotting detection reagents (GE Healthcare, Little Chalfont, UK).

For detection of phosphorylated YAP and TAZ, cell lysates were subjected to SDS-PAGE containing a phosphorylation tag (Phos-Tag ${ }^{\mathrm{Tx}}$, Wako, Osaka, Japan) that specifically traps phosphorylated proteins in the gel. After immunoblotting with anti-YAP or anti-TAZ IgG antibodies, phosphorylated proteins were detected as slower-migrating bands compared to the corresponding unphosphorylated proteins.

\section{Real-time polymerase chain reaction}

Total RNA from cultured C-MSCs was extracted using RNA-iso $^{\circ}$ (Takara, Otsu, Japan) and quantified by spectrometry at 260 and $280 \mathrm{~nm}$. RNA samples (500 ng) were reverse-transcribed into complementary DNA using ReverTra Ace (Toyobo, Osaka, Japan). Then, real-time PCR was performed using a LightCycler and SYBR green (Roche Applied Science, Mannheim, Germany) to determine the relative mRNA expression of collagen type I (COL1), connective tissue growth factor (CTGF), cysteine-rich angiogenic inducer 61 (CYR61), YAP, TAZ, runt-related transcription factor 2 (RUNX2), osteocalcin $(O C N)$, peroxisome proliferator-activated receptor $\gamma$ (PPARy), activating protein 2 (AP2), sex determining region Y-box 9 (SOX9), and Aggrecan. The PCR thermal profile consisted of an initial $10 \mathrm{~min}$ at $95^{\circ} \mathrm{C}$, followed by 40 cycles of $95^{\circ} \mathrm{C}$ for $15 \mathrm{~s}$ and $60^{\circ} \mathrm{C}$ for $1 \mathrm{~min}$. Fold changes of gene of interest were calculated with $\Delta \Delta \mathrm{Ct}$ method using ribosomal protein $18 \mathrm{~S}$ as reference control. The sequences of the primers used in this study are listed in Additional file 1: Table S2. 


\section{Statistical analysis}

Experiments were repeated three or four times, and the results are expressed as the means $\pm \mathrm{SD}$. Differences between parameters were tested for statistical significance using unpaired Student's $t$ tests or ANOVA. Values of $p<0.05$ or $p<0.01$ were considered significant.

\section{Results}

YAP/TAZ activity is downregulated in 3D-cultured C-MSCs For the generation of 3D-cultured C-MSCs, confluent MSCs treated with ascorbic acid for 4days were scratched using the tip of a micropipette (Fig. 1a). The MSC/ECM complexes were detached from the culture plate and exhibited a sheet-like morphology (Fig. 1b). After 1 day, this cellular sheet rolled up to form round cell clumps, termed C-MSCs. Immunofluorescence analysis demonstrated that C-MSCs were formed from COL1 produced by the MSCs during the culture period (Fig. 1b). During the C-MSC culture process, F-actin and YAP/TAZ expression patterns were assessed by confocal microscopy. F-actin was thick and abundant at the beginning of C-MSC culture; however, it became thinner and degraded over time, suggesting that F-actin depolymerization occurred in a time-dependent manner (Fig. 1c). In accordance with F-actin depolymerization, YAP/TAZ translocated to the cytoplasm (Fig. 1c, d), and their fluorescence signals were difficult to detect in cultured C-MSCs on day 5 (Fig. 1c). In addition, Western blotting demonstrated that the YAP/TAZ protein levels decreased dramatically on day 1 , and this reduction was time-dependent (Fig. 1e). In contrast, their mRNA expression levels were unchanged (Fig. 1f), suggesting that the downregulation of YAP/TAZ in C-MSCs may not be due to transcriptional reduction but instead to proteasomal degradation. It is widely known that phosphorylation of YAP/TAZ leads to their cytoplasmic retention and degradation, which results in the inhibition of YAP/ TAZ transcriptional activity. Therefore, next, we measured the phosphorylation levels of YAP/TAZ before their degradation. Notably, YAP/TAZ mobility shifts in a phos-tag gel, suggesting their phosphorylation, were induced in a time-dependent manner after $60 \mathrm{~min}$ of detachment (Fig. 1g). Consistent with these findings, the mRNA expression levels of the YAP/TAZ transcriptional target genes CTGF and CYR61 were also markedly decreased on day 1 , and the levels remained reduced until the end of the culture period (Fig. 1h). In contrast, MSCs maintained on 2D plastic culture plates kept abundant F-actin fiber structure and demonstrated only slight reductions in YAP/TAZ activity on day 5 (Additional file 2: Figure S2). These findings clearly indicated that YAP/TAZ activity is downregulated in association with actin degradation in 3D floating cultured C-MSCs.
3D-cultured C-MSCs can be directed towards adipogenesis and chondrogenesis, but not osteogenesis, because of reduced YAP/TAZ activity

Because it is well established that downregulation of $\mathrm{YAP} / \mathrm{TAZ}$ is responsible for adipogenesis/chondrogenesis but not osteogenesis in MSCs [21, 22], we hypothesized that C-MSCs, which showed decreased YAP/TAZ activity, could be directed towards adipo-/chondrogenesis. To test this hypothesis, various induction patterns were examined. OIM treatment caused a twofold increase in RUNX2 mRNA expression, although OCN mRNA levels were not affected by OIM (Fig. 2a). Furthermore, AIM or CIM dramatically increased PPARY and AP2 or Sox 9 and Aggrecan mRNA expression levels in C-MSCs, respectively (Fig. 2b, c). Dual induction, consisting of equal volumes of OIM and AIM, markedly elevated adipogenic marker genes but not osteogenic genes (Fig. 2d). Importantly, overexpression of a constitutively active form of TAZ (TAZS89A) significantly increased both RUNX2 and OCN mRNA expression levels in C-MSCs cultured with OIM (Fig. 2e). However, TAZS89A-transduced C-MSCs demonstrated decreased levels of adipogenic and chondrogenic marker gene expression with AIM and CIM treatment, respectively (Fig. 2f, g). These results indicated that 3D floating cultured C-MSCs are susceptible to adipo-/chondrogenic induction because of low YAP/TAZ activity.

However, it is possible that our primary MSCs are preferentially directed into adipo-/chondrogenesis instead of osteogenesis. To examine this possibility, we investigated the effects of each induction medium on cells cultured on 2D plastic culture plates at low cell densities were investigated. Consistent with previous studies [21], subconfluent MSCs on 2D culture plates showed high YAP/TAZ activity (Additional file 2: Figure S3A and S3B). Importantly, 2D-cultured cells demonstrated remarkable increases in osteogenesis but only slight or insufficient adipogenesis and chondrogenesis (Additional file 2: Figure S3C-S3F). In addition, knockdown of YAP/TAZ by siRNA transfection reduced osteogenesis in 2D-cultured MSCs, complementing increases in adipogenesis and chondrogenesis (Additional file 2: Figure S3H-S3J). These findings indicate that our cells possess osteogenic properties in culture conditions with increased YAP/TAZ activity. Therefore, the adipo-/chondrogenic properties of 3D-cultured C-MSCs are due to reduced YAP/TAZ activity.

\section{OIM facilitates F-actin formation and YAP/TAZ activity in C-MSCs}

Previously, we reported that OIM-C-MSCs (C-MSCs cultured with OIM for 5 days) exert more effective bone regenerative properties in vivo than C-MSCs $[4,6]$. Moreover, OIM-C-MSCs showed slightly but significantly increased $R U N X 2$ mRNA expression, as shown in 

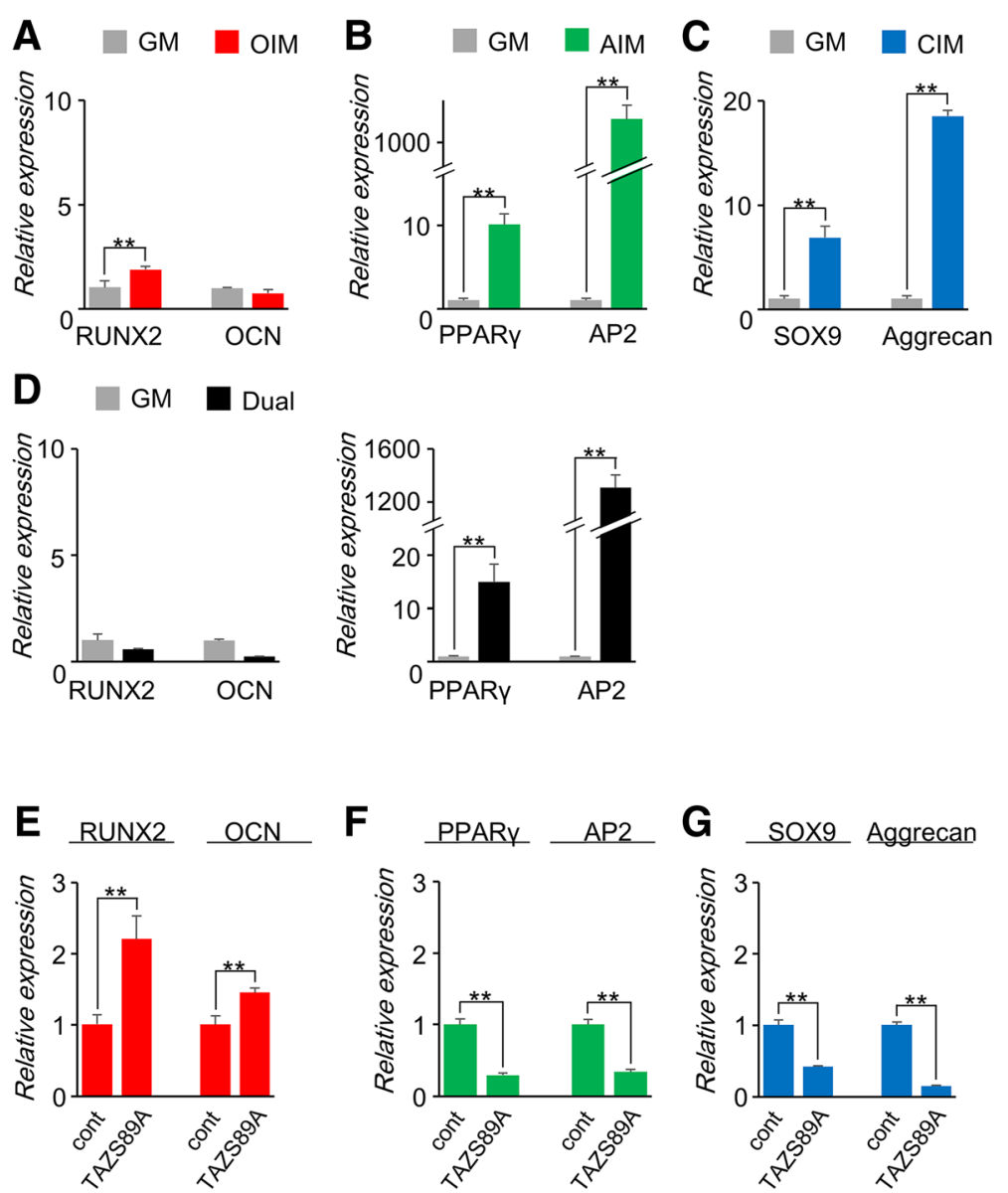

Fig. 2 Three-dimensional floating culture C-MSCs can be directed towards adipogenesis/chondrogenesis but not osteogenesis due to reduced YAP/TAZ activity. a-d C-MSCs were cultured in a OIM, b AIM, c CIM, or $\mathbf{d}$ dual medium for 5 days. Differentiation marker gene expression levels were analyzed by real-time PCR. Data were normalized to the values of C-MSCs maintained in GM. $\mathbf{e}-\mathbf{g}$ C-MSCs transfected with a constitutively active TAZ mutant (TAZS89A) or control plasmid were maintained in e OIM, f AIM, or $\mathbf{g}$ CIM for 5 days. Differentiation marker gene expression levels were analyzed by real-time PCR. Data were normalized to the values of C-MSCs transfected with control vector (cont). Values represent means \pm S.D. of three cultures $\left({ }^{* *} p<0.01\right)$. All graphs are representative of three independent experiments

Fig. 2a, indicating that the biological characteristics of OIM-C-MSCs could be more osteogenic than those of C-MSCs. Importantly, because it is widely accepted that high YAP/TAZ activity is responsible for osteogenesis in MSCs [21, 25], we next hypothesized that OIM treatment ameliorates YAP/TAZ activity in 3D floating cultured $\mathrm{C}$-MSCs to induce osteogenesis. First, we determined that OIM treatment increased COL1 expression, which induces C-MSC morphology in a time-dependent manner (Fig. 3a). Importantly, unlike the YAP/TAZ and F-actin expression patterns in the C-MSCs described above (Fig. 1), C-MSCs treated with OIM maintained thick, abundant F-actin fibers, and YAP/TAZ expression for 5 days (Fig. 3b). In addition, OIM treatment restored the level of nuclear YAP/TAZ in C-MSCs, which was decreased after detachment for 1 day (Fig. 3b, c). Compared to C-MSCs, OIM-C-MSCs showed higher YAP/TAZ levels (Fig. 3d) and significantly increased YAP/TAZ transcriptional activity (Fig. 3e). Therefore, YAP/TAZ siRNAs were used to determine whether this elevated YAP/TAZ activity was associated with RUNX2 mRNA expression in OIM-C-MSCs. Co-transfection of YAP/TAZ siRNAs significantly decreased RUNX2 mRNA expression levels in OIM-C-MSCs (Fig. 3f). These findings indicated that OIM treatment facilitates YAP/TAZ activity to induce RUNX2 mRNA expression in 3D floating cultured C-MSCs.

COL1 deposition caused by OIM facilitates a mechanotransduction cascade composed of Int $\beta 1$-rhoassociated protein kinase (ROCK)-F-actin-YAP/TAZ signaling to induce osteogenesis in C-MSCs

To clarify how OIM elevates YAP/TAZ activity in C-MSCs, we investigated the effect of each component in OIM, including $\beta$-glycerophosphate (Gly), ascorbic acid (AA), and dexamethasone (Dex), on COL1 


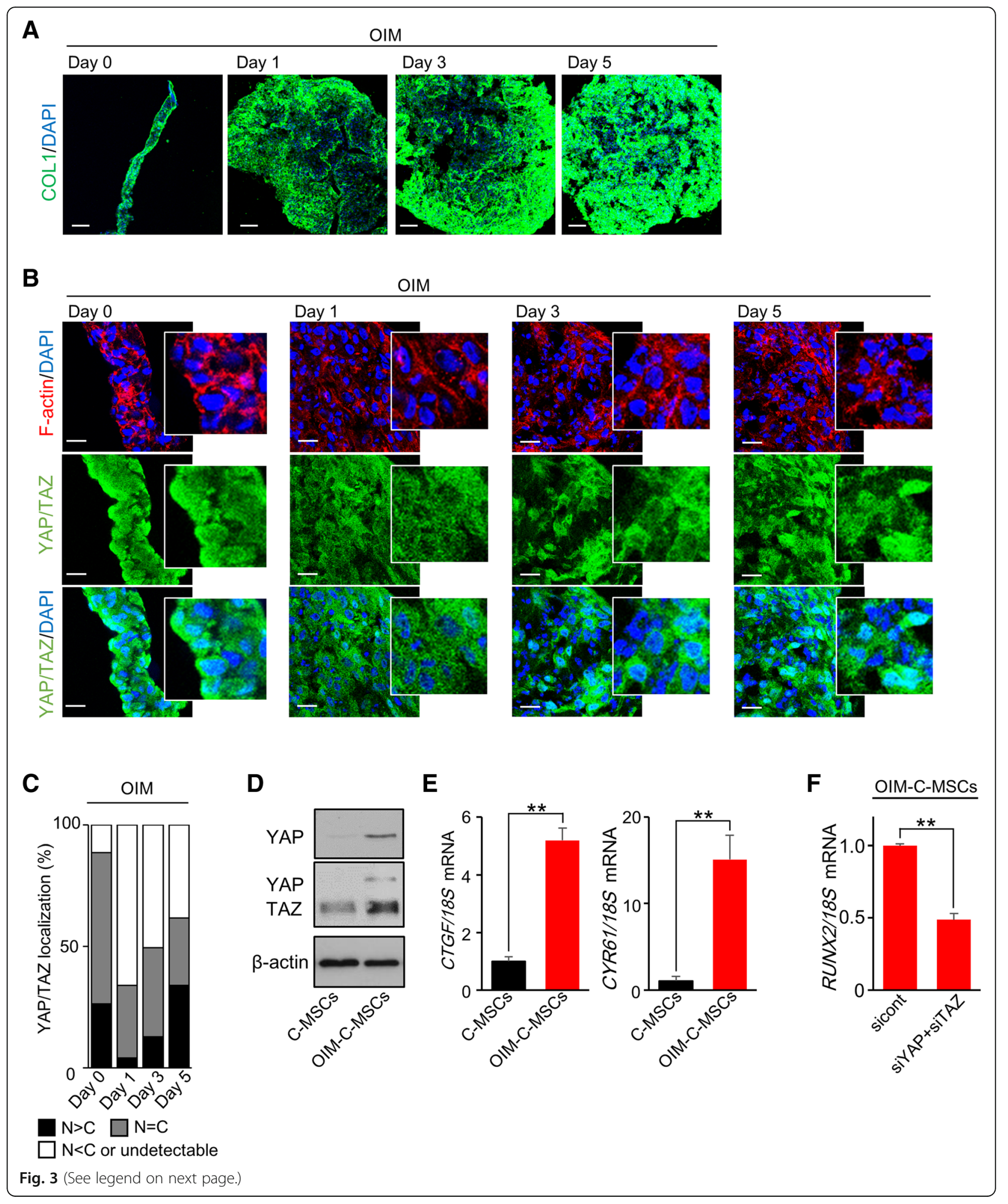


(See figure on previous page.)

Fig. 3 Osteoinductive medium facilitates F-actin formation and YAP/TAZ activity in C-MSCs. a, b C-MSCs were cultured in OIM for the indicated culture period. a Confocal immunofluorescence images of COL1 and nuclei. Bar $=100 \mu \mathrm{m}$. b Confocal immunofluorescence images of YAP/TAZ (green), F-actin (red), and nuclei (blue) in C-MSCs. Bar $=20 \mu \mathrm{m}$. White boxes show enlarged images. $\mathbf{c}$ The graph summarizes the distribution of YAP/TAZ localization patterns. The patterns were classified as mainly nuclear $(N>C)$, diffuse $(N=C)$, or mainly cytoplasmic or undetectable ( $\mathrm{N}<\mathrm{C}$ or undetectable). $\mathbf{d}-\mathbf{f}$ C-MSCs: C-MSCs cultured in GM for 5 days. OIM-C-MSCs: C-MSCs cultured in OIM for 5 days. $\mathbf{d}$ Immunoblotting for YAP/TAZ. A rabbit anti-YAP/TAZ (D24E4) mAb (Cell Signaling) was used to detect both the YAP and TAZ proteins (middle panel). To show YAP expression more clearly, we also used a rabbit anti-YAP (D8H1X) mAb (upper panel). e Real-time PCR of YAP/TAZ target genes. Data were normalized to the values of C-MSCs. Values represent means \pm S.D. of three cultures $\left({ }^{* *} p<0.01\right)$. $\mathbf{f}$ C-MSCs transfected with negative control or YAP and TAZ siRNAs were cultured in OIM for 5 days. RUNX2 expression levels were analyzed by real-time PCR. Data were normalized to the values of OIM-C-MSCS transfected with negative control siRNA. Values represent means \pm S.D. of three cultures (** $p<0.01$ ). All graphs and images are representative of three independent experiments

production, F-actin expression, and YAP/TAZ localization in C-MSCs. Immunofluorescence staining demonstrated that Dex- or Gly+Dex-treated groups showed higher COL1 production, F-actin formation, and increased YAP/ TAZ activity than the non-(GM only), Gly-, AA-, or Gly +AA-treated groups (Fig. 4a-c). Moreover, both AA+Dex and Gly+AA+Dex (OIM) treatment demonstrated the greatest amounts of COL1 deposition in C-MSCs (Fig. 4a). Consistent with this increased COL1 production, F-actin integrity and YAP/TAZ activity were also strongest in all tested groups (Fig. 4b, c). These findings suggested the possibility that COL1 deposition caused by OIM (mainly the Dex and AA components) plays a role in the upregulation of F-actin formation and YAP/TAZ activity.

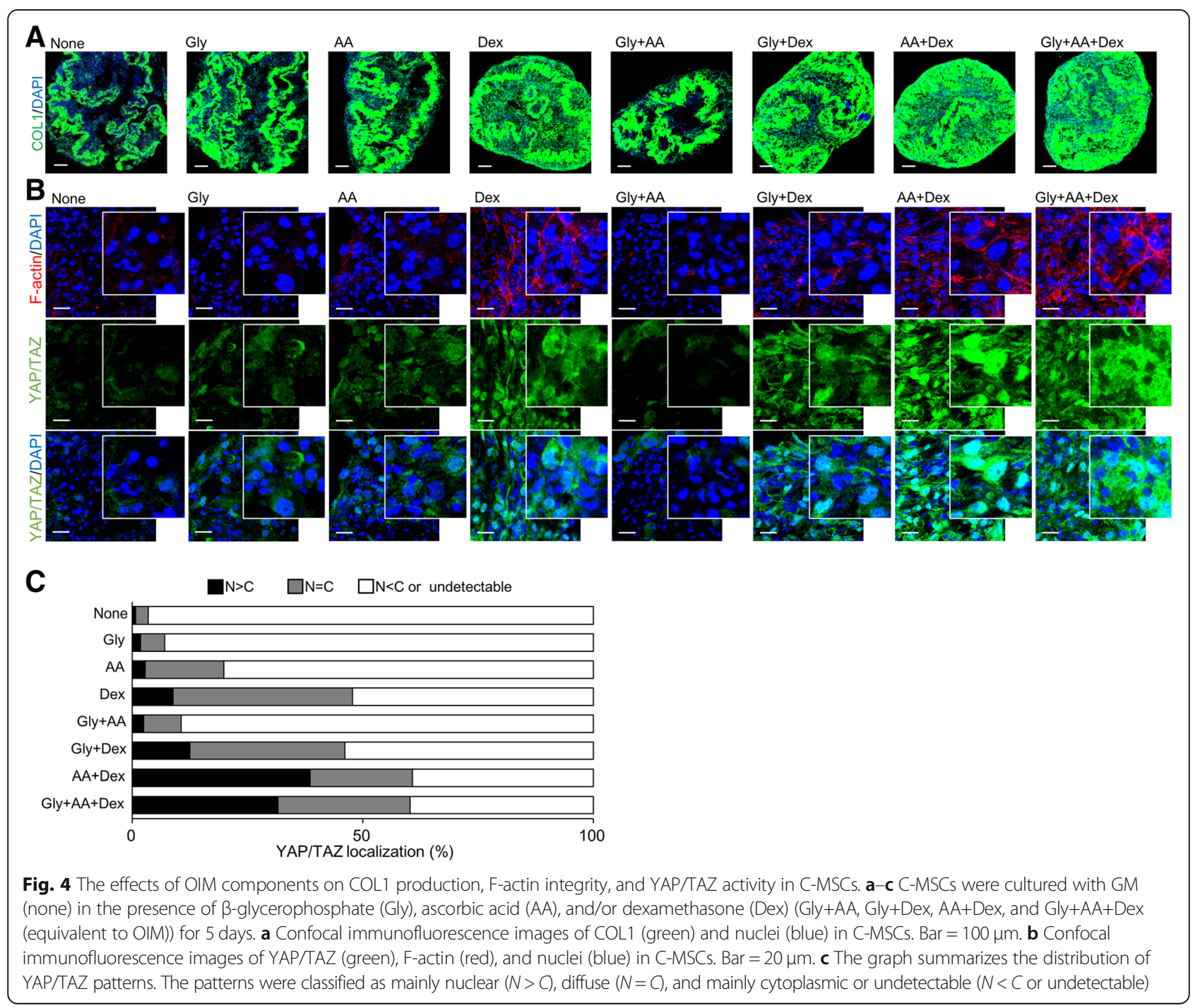


Accordingly, we investigated the effects of Int $\beta 1$ siRNA on YAP/TAZ activity in OIM-C-MSCs because Int $\beta 1$ links the ECM, including COL1, with the actin cytoskeleton, thereby transmitting signals from the ECM to the cytoplasm [26]. Notably, OIM-C-MSCs transfected with Int $\beta 1$ siRNA showed disruption of F-actin fibers (Fig. 5a). Importantly, knockdown of Int $\beta 1$ attenuated YAP/TAZ expression, and undegraded YAP/TAZ was localized mainly in the cytoplasm of OIM-C-MSCs (Fig. 5a, b). Western blotting demonstrated that Int $\beta 1$ siRNA treatment decreased YAP/TAZ expression levels in OIM-C-MSCs (Fig. $5 \mathrm{c}$ ). Depletion of Int $\beta 1$ by siRNA abrogated CTGF and CYR61 mRNA expression levels, suggesting the suppression of YAP/TAZ transcriptional activity (Fig. 5d). In addition, OIM-C-MSCs transfected with Int $\beta 1$ siRNA showed decreased expression levels of RUNX2 mRNA (Fig. 5e). These findings indicated that OIM-induced COL1 could increase F-actin integrity and YAP/TAZ activity via Int $\beta 1$ signaling in $3 \mathrm{D}$-cultured C-MSCs.

Because ROCK is well established as a downstream target of Int $\beta 1$, which facilitates actin polymerization to induce traction force, we next explored the relationship between OIM-induced YAP/TAZ activity and ROCK or the actin cytoskeletal tension using chemical inhibitors. Inhibition of ROCK activity by Y27632 treatment drastically diminished F-actin fibers, consistent with reductions in YAP/TAZ expression levels in OIM-C-MSCs (Fig. 5f). OIM-C-MSCs treated with blebbistatin showed disruption of actin fiber integrity, suggesting a reduction in cytoskeletal tension and degradation of YAP/TAZ (Fig. 5f). YAP/TAZ that had avoided degradation in both Y27632- and blebbistatin-treated groups were mainly localized in the cytoplasm (Fig. 5f, g). Western blotting also showed downregulation of YAP/TAZ expression in OIM-C-MSCs treated with Y27632 and blebbistatin (Fig. 5h). Real-time PCR demonstrated that the YAP/TAZ target genes CTGF and CYR61 were reduced by treatment with these chemical inhibitors as well (Fig. 5i). In addition, RUNX2 mRNA expression levels in OIM-C-MSCs were reduced by ROCK inhibition and F-actin disruption (Fig. 5j). These findings suggested that OIM-induced YAP/TAZ activity was dependent on F-actin fiber integrity regulation by ROCK in 3D-cultured C-MSCs.

YAP/TAZ activity is associated with COL1 synthesis and F-actin formation, generating a positive feedback loop in OIM-C-MSCs

CTGF, which was upregulated via YAP/TAZ activity in OIM-C-MSCs, can induce COL1 production in MSCs [27]. Accordingly, we hypothesized that upregulated YAP/ TAZ activity via OIM treatment was associated with COL1 synthesis, thereby resulting in the activation of a positive feedback mechanotransduction loop in OIM-CMSCs. Depletion of YAP/TAZ by siRNA co-transfection significantly decreased COL1A1 mRNA and protein expression levels in OIM-C-MSCs (Fig. 6a, b). Notably, YAP/TAZ siRNA treatment abolished not only YAP/TAZ but also F-actin fibers in OIM-C-MSCs (Fig. 6c). Moreover, Int $\beta 1$ siRNA, which reduced F-actin integrity and YAP/TAZ activity in OIM-C-MSCs (Fig. 5a-d), significantly decreased COL1A1 mRNA and protein expression levels (Fig. 6d, e). Similarly, inhibition of ROCK and non-muscle acto-myosin using Y27632 and blebbistatin, which disrupted F-actin fibers and YAP/TAZ activity in OIM-C-MSCs (Fig. 5f-i), also clearly reduced COL1 gene and protein expression levels, respectively (Fig. 6f, g). These findings suggested that YAP/TAZ activity caused by OIM treatment can induce COL1 production to activate a positive feedback mechanotransduction loop, which includes Int 1 1-ROCK-F-actin formation, in C-MSCs. To support these findings, we examined the effects of constitutively active TAZS89A overexpression on COL1 production, F-actin polymerization, and YAP/TAZ expression levels in C-MSCs, which resulted in decreased YAP/TAZ activity (Fig. 1). TAZS89A-transduced C-MSCs demonstrated increased levels of COL1A1 mRNA and protein expression (Fig. 6h, i). TAZS89A dramatically stimulated F-actin formation consistent with the elevation of YAP/ TAZ/TAZS89A expression and nuclear localization (Fig. 6j). Western blotting analysis demonstrated that transfection of TAZS89A increased not only TAZ/ TAZS89A expression but also YAP expression levels in C-MSCs (Fig. 6k). Real-time PCR analysis demonstrated that TAZ, CTGF, and CYR61 mRNA expression levels were significantly increased by TAZS89A transfection, although YAP mRNA expression levels were not affected (Fig. 6l). These findings suggested that the elevated YAP protein levels in Fig. $6 \mathrm{k}$ were not caused by TAZS89A transcriptional activity but rather by the suppression of proteasomal degradation. Notably, treatment with the actin-disrupting drugs Y27632 and blebbistatin decreased COL1 production and YAP/TAZ activity induced by TAZS89A transfection (Additional file 2: Figure S4). Taken together, these findings showed the presence of a self-reinforcing feed-forward loop between the matrix and YAP/TAZ linked by cytoskeletal mechanisms, similar to the positive feedback regulation described for C-MSCs treated with OIM.

\section{Discussion}

This present study demonstrated that MSCs 2D monolayers detached from the culture dish form 3D structures where YAP/TAZ are inactivated keeping with mechano-regulation of these factors and that culturing floating monolayers in OIM is sufficient to install a feed-forward loop between COL1 production, integrin engagement, acto-myosin contractility, YAP/TAZ activity, which in turn further amplifies COL1 transcription 


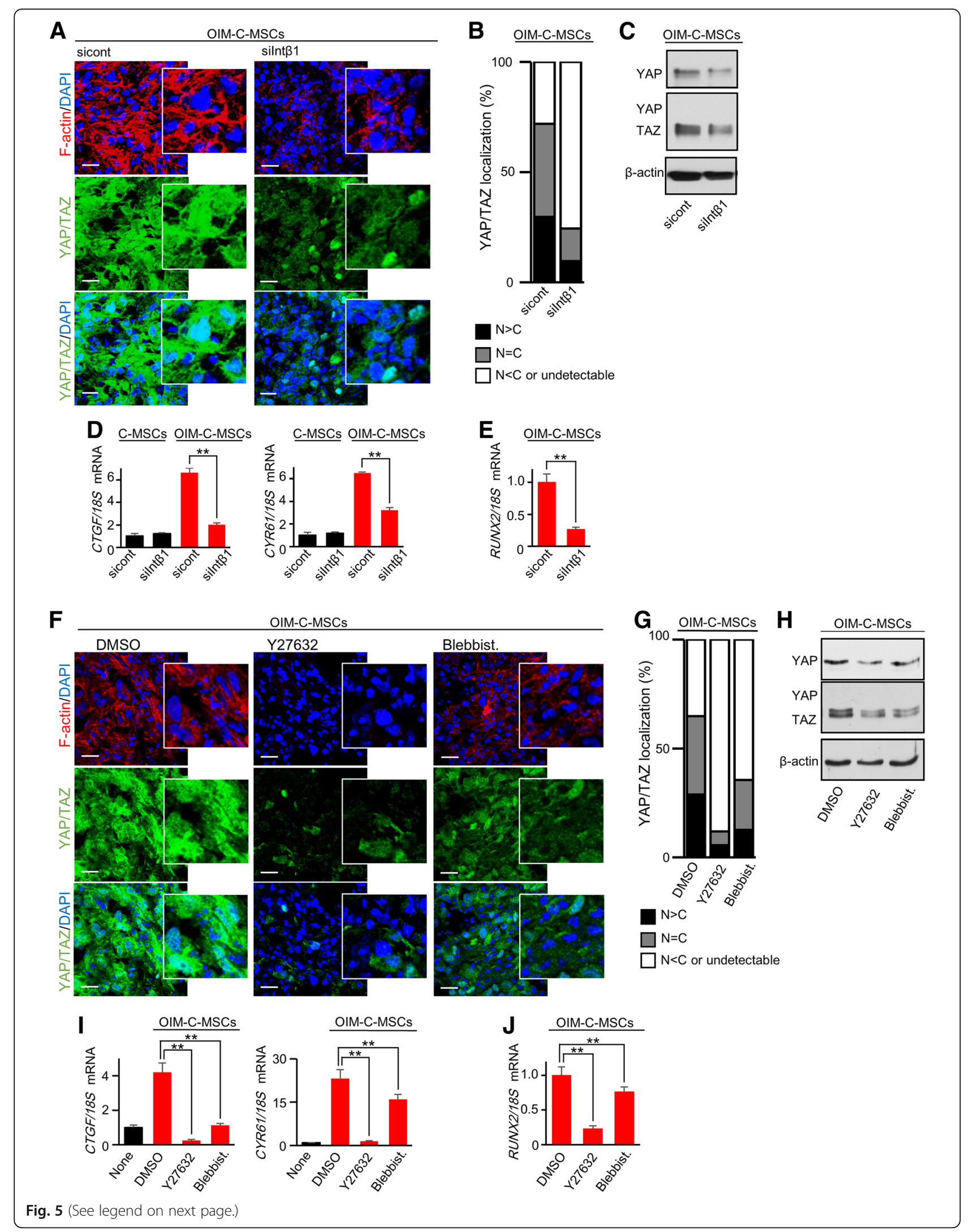


(See figure on previous page.)

Fig. 5 OIM-induced YAP/TAZ activity in C-MSCs is due to actin cytoskeletal tension caused by Int $\beta 1$-ROCK signaling. a-j C-MSCs: C-MSCs cultured with GM for 5 days. OIM-C-MSCs: C-MSCs cultured with OIM for 5 days. a-e C-MSCs transfected with negative control or Int $\beta 1$ siRNAs were cultured with GM or OIM for 5 days. a Confocal immunofluorescence images of YAP/TAZ (green), F-actin (red), and nuclei (blue) in OIM-C-MSCs. $\mathrm{Bar}=20 \mu \mathrm{m}$. White boxes show enlarged images. b The graph summarizes the distribution of YAP/TAZ localization patterns. The patterns were classified as mainly nuclear ( $N>C$ ), diffuse ( $N=C$ ), and mainly cytoplasmic or undetectable ( $N<C$ or undetectable). c Immunoblotting for YAP/ TAZ in OIM-C-MSCs. A rabbit anti-YAP/TAZ (D24E4) mAb (Cell Signaling) was used to detect both the YAP and TAZ proteins (middle panel). To show YAP expression more clearly, we also used a rabbit anti-YAP (D8H1X) mAb (upper panel). d Real-time PCR analysis of YAP/TAZ target genes. Data were normalized to the values of control siRNA-transfected C-MSCs. Values represent means \pm S.D. of three cultures (** $p<0.01)$. e RUNX2 expression levels in OIM-C-MSCs were analyzed by real-time PCR. Data were normalized to the values of OIM-C-MSCs transfected with negative control siRNA. Values represent means \pm S.D. of three cultures $\left({ }^{* *} p<0.01\right)$. All graphs and images are representative of three independent experiments. f-j C-MSCs or OIM-C-MSCs were cultured with or without a ROCK inhibitor (Y27632, $50 \mu \mathrm{M})$, the non-muscle myosin inhibitor blebbistatin (Blebbist., $50 \mu \mathrm{M}$ ), or an appropriate concentration of DMSO for 5 days. $\mathbf{f}$ Confocal immunofluorescence images of YAP/TAZ (green), F-actin (red), and nuclei (blue) in OIM-C-MSCs. Bar = $20 \mu \mathrm{m}$. White boxes show enlarged images. $\mathbf{g}$ The graph summarizes the distribution of YAP/TAZ localization patterns. The patterns were classified as mainly nuclear ( $N>C$ ), diffuse $(N=C)$, and mainly cytoplasmic or undetectable ( $<C$ or undetectable). $\mathbf{h}$ Immunoblotting for YAP/TAZ in OIM-C-MSCs. A rabbit anti-YAP/TAZ (D24E4) mAb (Cell Signaling) was used to detect both the YAP and TAZ proteins (middle panel). To show YAP expression more clearly, we also used a rabbit anti-YAP (D8H1X) mAb (upper panel). i Real-time PCR analysis of YAP/TAZ target genes. Data were normalized to the values of C-MSCs treated with DMSO. Values represent means \pm S.D. of three cultures $\left({ }^{* *} p<0.01\right)$. $\mathbf{j} R U N X 2$ expression levels in OIM-C-MSCS were analyzed by real-time PCR. Data were normalized to the values of OIM-C-MSCs treated with DMSO. Values represent means \pm S.D. of three cultures $\left.*^{* *} p<0.01\right)$. All graphs and images are representative of three independent experiments

(Fig. 7). These findings indicate for the first time that ECM molecules produced by cell clumps growing in suspension can be sufficient to keep cytoskeletal contractility and YAP/TAZ active. This fact may have broad relevance for the other famous $3 \mathrm{D}$ cell construct, such as cell spheroid [28-30] or organoid [31, 32], which are also cultured with suspension condition. Indeed, recent study reported that OIM-pretreated MSC spheroids retain the osteogenic phenotype due to Int $\alpha 2 \beta 1$ engagement with the cell-secreted extracellular matrix [33], implying the existence of ECM-mediated YAP/TAZ positive feedback loop. Moreover, Takebe et al. demonstrated that MSC-driven cytoskeletal contraction is indispensable for dynamic formation of various type of organoids [34]. Therefore, this present study may shed light on the key mechano-regulators of 3D cell constructs, which have attracted scientific and medical attention for promising tissue regenerative therapy.

The dominant mechanical cue that occurred during the generation of C-MSCs was the detachment of the cellular sheet from the culture plate. F-actin fibers and YAP/TAZ degradation continued until the end of the C-MSC floating culture period (Fig. 1). In these suspended conditions, it could be challenging to develop sufficient cytoskeletal tension to elevate YAP/TAZ activity in C-MSCs. These findings may suggest that chondrogenic induction of MSCs in vitro preferentially occurs in floating spheroidal culture systems rather than conventional 2D culture on a plastic plate [35]. Because chondrogenesis is abolished by high YAP/TAZ activity [36], the activity in cells attached to a stiff substrate could be too high to induce successful chondrogenesis.

It has been shown that cell detachment from the culture plate or ECM induced a specific type of apoptosis, termed anoikis. Notably, a recent study demonstrated that the loss of attachment by trypsinization reduced cytoskeletal tension to decrease YAP activity, which resulted in the induction of anoikis [23]. Even though this present study also indicated similar mechanotransduction, few apoptotic cells were observed in floating C-MSCs, as described previously [5]. Because C-MSCs consisted of self-produced ECM that could provide the cells with adhesion conditions, compared with single-cell suspension, YAP/TAZ activity in C-MSCs could be maintained at a slightly higher level to escape anoikis.

OIM, which consists of Dex, AA, and Gly, is a frequently employed culture method for tissue engineering approaches or assessment of the osteogenic differentiation capacity of various cell types [37]. Indeed, OIM has been used to demonstrate the relationship between YAP/TAZ signaling and osteogenesis in vitro [22, 38]. However, few studies documenting the direct effects of OIM on YAP/TAZ activity have been reported. This present study demonstrated that OIM stimulated YAP/TAZ activity to increase RUNX2 mRNA expression in C-MSCs. The key signaling event to induce such YAP/TAZ activity is dependent on an Int $\beta 1$-ROCK-F-actin mechanotransduction pathway generated by OIM-induced COL1 deposition. More specifically, we demonstrated that, among the OIM components, the combination of Dex and AA plays a role in COL1 production in association with elevated YAP/TAZ activity in C-MSCs (Fig. 4). Dex upregulates COL1A1 mRNA expression levels in murine and human MSCs [39-42]. AA increases COL1 mRNA stability [43] and upregulates secretion of COL1 by inducing proper collagen-triple helix formation [44]. Accordingly, although the precise interaction mechanism of the single elements in OIM is still unclear, Dex and AA may increase COL1 deposition in a coordinated manner to stimulate the YAP/TAZ mechanotransduction pathway in C-MSCs. 


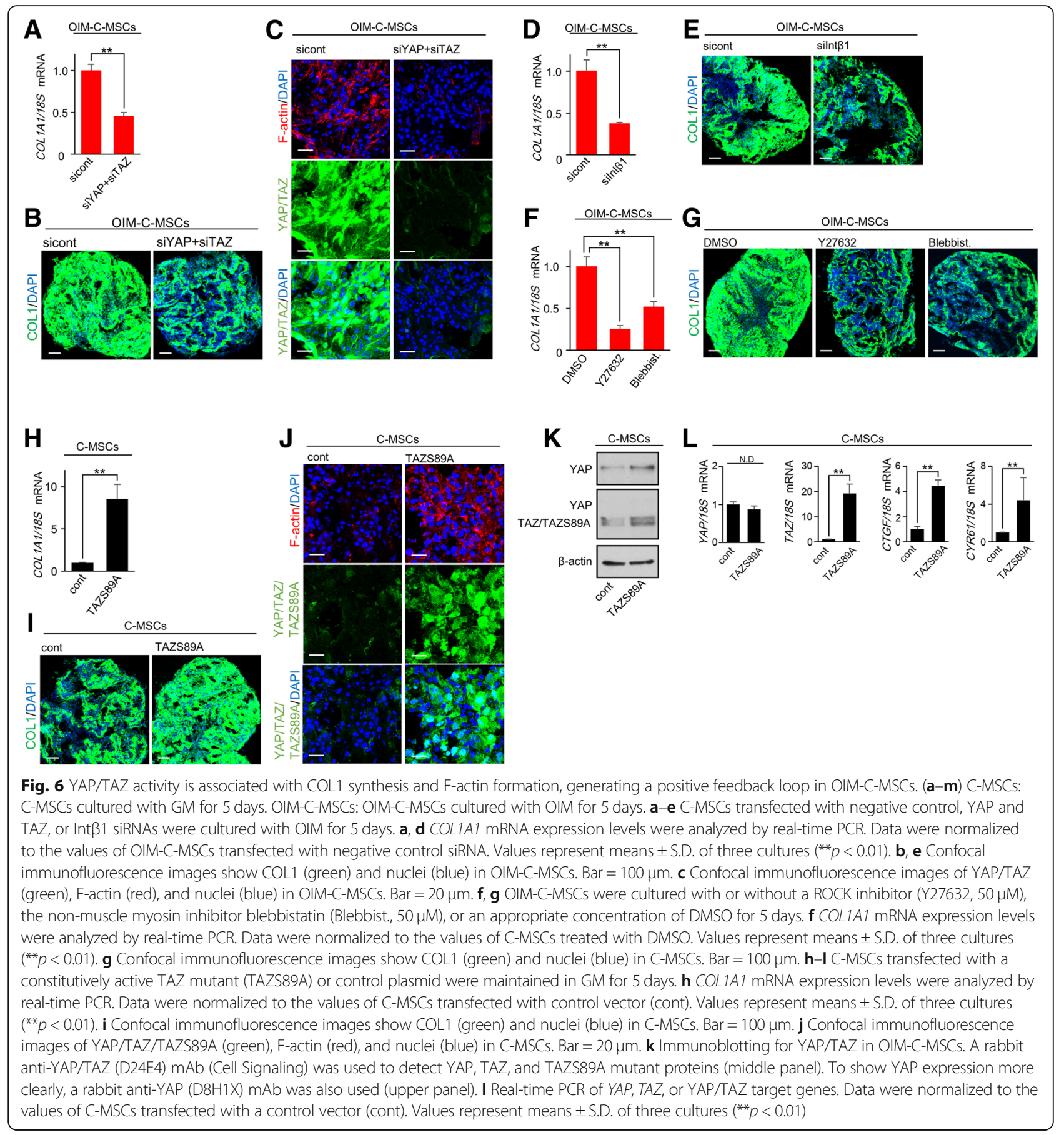

The present study revealed that abundant COL1 deposition in OIM-C-MSCs formed a positive feedback loop for YAP/TAZ-regulated mechanotransduction, which was primarily maintained by cytoskeletal tension. In addition, overexpression of TAZS89A also induced a similar feed-forward loop promoting YAP/TAZ activity in C-MSCs. Recently, this type of feed-forward loop of ECM and cytoskeletal tension has been reported in various types of diseases, including cancer [45], lung fibrosis [46], liver fibrosis [47], and pulmonary hypertension [48, 49]. Although aberrant mechanical signaling by YAP/TAZ is associated with the pathogenesis of multiple diseases, YAP/TAZ signaling is also responsible for normal development, organ growth, and tissue regeneration. Therefore, this emerging positive feedback loop of YAP/TAZ may play a role in regulating biological functions in living organisms.

Notably, in contrast to the present study, constitutive active TAZ4SA overexpression in a NIH3T3 mouse 

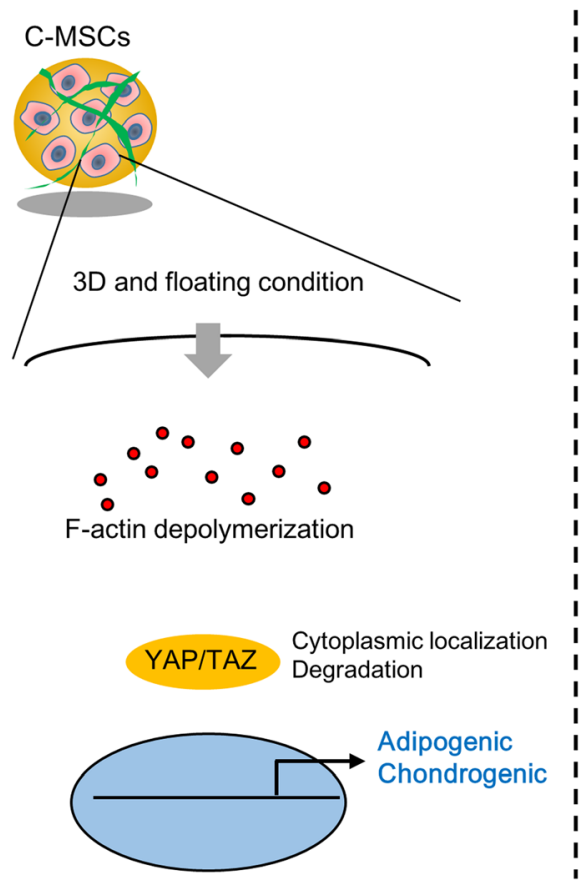

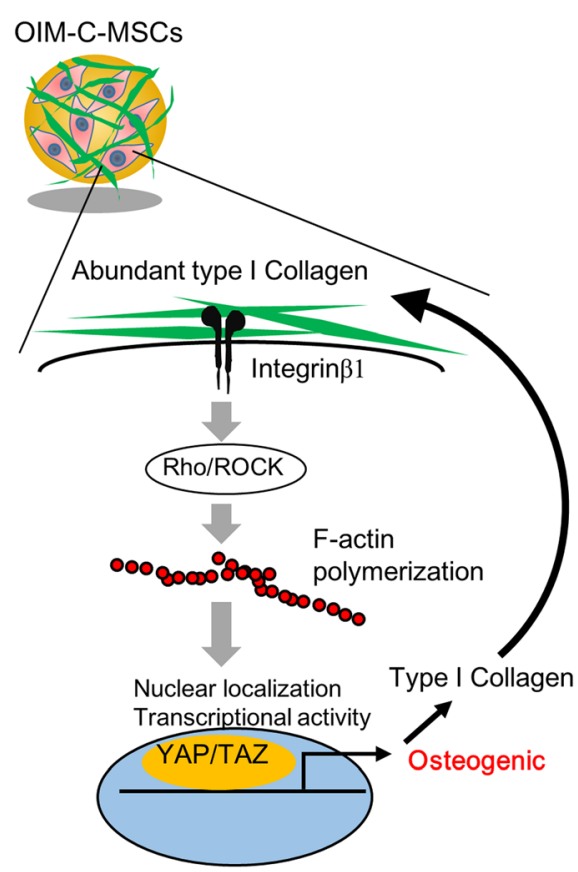

Fig. 7 Schematic summary of mechanotransduction cascade in C-MSCs and OIM-C-MSCs

fibroblast cell line did not induce ECM deposition or produce a positive feedback loop in other scaffold-free culture systems, i.e., spheroid culture, even though TAZ activity was elevated [50]. The fibroblast spheroids consisted of cells that organize themselves via cell-cell contact. However, no ECM expression was detected in the spheroids by histological analysis. Accordingly, the conflicting findings regarding positive feedback loop formation by transfection of a constitutive active TAZ mutant between C-MSCs and fibroblast spheroids may imply that a certain threshold level of ECM deposition, cytoskeletal tension, and YAP/TAZ activity is essential to generate the feed-forward loop.

Regarding the clinical application of C-MSCs or OIM-C-MSCs for bone regenerative therapy, we reported previously that transplantation of OIM-C-MSCs exerted robust, rapid new bone formation in a bone defect site compared to C-MSCs $[4,6]$. This present in vitro study may explain our previous findings. First, C-MSCs, which showed low YAP/TAZ activity, were preferentially induced into adipocytes/chondrocytes but not osteocytes. Second, OIM-C-MSCs showed high YAP/TAZ activity and increased RUNX2 mRNA expression, which are well-known transcription factors for osteogenic differentiation [51]. Third, OIM-C-MSC transplantation can provide abundant COL1, which has been recognized as an indispensable ECM protein for new bone formation, at a bone defect site [52]. Finally, a positive feedback loop involving COL1 deposition and YAP/TAZ activation may accelerate the bone regenerative properties of OIM-C-MSCs after transplantation. A future study investigating these molecular mechanisms in vivo may provide additional support for novel bone regenerative cell therapies using 3D-cultured C-MSCs.

In this present study, we mainly focused on the cellular property in 5-day OIM-treated C-MSCs. Because the 10-day or 15-day OIM-treated C-MSCs failed to induce bone regeneration in vivo $[4,6]$. Interestingly, OIM treatment for more than 10 days induced mineralization, though profound cell apoptosis was also observed in C-MSCs [4, 6]. These findings suggested that excess osteoinduction in vitro may induce C-MSCs to become cellular calcific substances, not biological bone-like tissue. Although the precise molecular mechanism is unclear, mineral deposition may be toxic to the cells in the floating condition which decreased YAP/TAZ activity. To develop the culture protocol that can abrogate the mineral deposition-induced cell apoptosis in floating condition will provide us the biological bone-like tissue in vitro by using C-MSCs.

\section{Conclusion}

This present study clearly demonstrated the distinction of biological capacities between C-MSCs and OIM-CMSCs in the context of mechanobiology. Briefly, 3D-cultured C-MSCs in floating conditions lost their actin cytoskeleton and had decreased YAP/TAZ activity, which directed cells to undergo adipo-/chondrogenesis. In contrast, OIM treatment facilitated COL1 deposition, which stimulated Int $\beta 1-R O C K-F-a c t i n-Y A P / T A Z$ 
signaling to induce osteogenesis in C-MSCs. Importantly, the mechanotransduction cascade induced by OIM generated a positive feedback loop of YAP/TAZ activity in C-MSCs. These findings suggest that OIM-CMSCs, which form a microenvironment that maintains high YAP/TAZ activity, are a superior candidate cell type for bone regenerative cell therapy.

\section{Additional files}

Additional file 1: Table S1. Sequences of siRNA oligonucleotides. Table S2. Sense and antisense primers for real-time PCR (DOCX 29 kb)

Additional file 2: Figure S1. Effect of siRNAs or DNA expression plasmids transfections in C-MSCs. Figure S2. MSCs cultured on 2D plastic culture plate maintained YAP/TAZ activity. Figure S3. High YAP/TAZ activity in subconfluent MSCs cultured on a 2D plastic plate regulates the cell lineage into osteogenesis but not adipo/chondrogenesis. Figure S4. Disruption of F-actin integrity by ROCK inhibitor and acto-myosin inhibitor abrogates the TAZS89A-induced positive feedback loop for in C-MSCs. (ZIP $6647 \mathrm{~kb})$

\section{Abbreviations}

2D/3D: 2/3-Dimensional; AlM: Adipogenic induction medium; AP-2: Activating protein 2; CIM: Chondrogenic induction medium; C-MSCs: Clumps of mesenchymal stem cells/extracellular matrix proteins; COL1: Collagen type l: CTGF: Connective tissue growth factor; CYR61: Cysteine-rich angiogenic inducer 61; DAPI: 4'6-Diamidino-2-phenylindole; DMEM: Dulbecco's modified Eagle's medium; DMSO: Dimethyl sulfoxide; ECM: Extracellular matrix; Factin: Filamentous actin; FBS: Fetal bovine serum; Int $\beta 1$ : Integrin $\beta 1$; MSCs: Mesenchymal stem cells; OCN: Osteocalcin; OIM: Osteogenic induction medium; PPARY: Peroxisome proliferator-activated receptor $\gamma ;$ ROCK: Rhoassociated protein kinase; RUNX2: Runt-related transcription factor 2; Sox9: SRY (sex determining region Y)-box 9; TAZ: Transcriptional co-activator with PDZbinding motif; TEAD: TEA transcriptional factor; YAP: Yes-associated protein

\section{Acknowledgements}

We thank Daniel Mrozek who provided English language editing services on behalf of Medical English Service (Kyoto, Japan).

\section{Funding}

This work was supported by JSPS KAKENHI Grant Numbers JP18H02977, JP17K173510A, and JP17H06897 and a Research Grant from The Nakatomi Foundation and Mitsui Sumitomo Insurance Welfare Foundation.

\section{Availability of data and materials}

The datasets used or analyzed during the current study available from the corresponding author on reasonable request.

\section{Authors' contributions}

NK contributed to the study conception and design, collection and assembly of data, data analysis and interpretation, manuscript writing, and final approval of manuscript. MK contributed to the study conception and design, financial support, collection and assembly of data, data analysis and interpretation, manuscript writing, and final approval of manuscript. SM, MT, SH, Tl, and $\mathrm{KO}$ contributed to the collection and assembly of data and final approval of manuscript. KT, SM, and TF contributed to the provision of study material, data analysis and interpretation, and final approval of manuscript. HK contributed to the study conception and design, financial support, administrative support, manuscript writing, and final approval of manuscript.

\section{Ethics approval and consent to participate}

Ethical approval for human subjects was obtained from the Independent Ethics Committee of Hiroshima University (reference number 422-2).

\section{Consent for publication}

Not applicable. This manuscript does not contain any individual person's data.

\section{Competing interests}

The authors declare that they have no competing interests.

\section{Publisher's Note}

Springer Nature remains neutral with regard to jurisdictional claims in published maps and institutional affiliations.

Received: 25 September 2018 Revised: 10 November 2018 Accepted: 20 November 2018 Published online: 07 December 2018

\section{References}

1. Samsonraj RM, Raghunath M, Nurcombe V, Hui JH, van Wijnen AJ, Cool SM. Concise review: multifaceted characterization of human mesenchymal stem cells for use in regenerative medicine. Stem Cells Transl Med. 2017;6(12): 2173-85.

2. Bose S, Roy M, Bandyopadhyay A. Recent advances in bone tissue engineering scaffolds. Trends Biotechnol. 2012;30(10):546-54.

3. Nagahara T, Yoshimatsu S, Shiba H, Kawaguchi H, Takeda K, Iwata T, Mizuno $\mathrm{N}$, Fujita T, Kurihara $\mathrm{H}$. Introduction of a mixture of beta-tricalcium phosphate into a complex of bone marrow mesenchymal stem cells and type I collagen can augment the volume of alveolar bone without impairing cementum regeneration. J Periodontol. 2015;86(3):456-64.

4. Kittaka M, Kajiya M, Shiba H, Takewaki M, Takeshita K, Khung R, Fujita T, Iwata T, Nguyen TQ, Ouhara K, et al. Clumps of a mesenchymal stromal cell/ extracellular matrix complex can be a novel tissue engineering therapy for bone regeneration. Cytotherapy. 2015;17(7):860-73.

5. Takeshita K, Motoike S, Kajiya M, Komatsu N, Takewaki M, Ouhara K, Iwata T, Takeda K, Mizuno N, Fujita T, et al. Xenotransplantation of interferongamma-pretreated clumps of a human mesenchymal stem cell/extracellular matrix complex induces mouse calvarial bone regeneration. Stem Cell Res Ther. 2017;8(1):101.

6. Takewaki M, Kajiya M, Takeda K, Sasaki S, Motoike S, Komatsu N, Matsuda S, Ouhara K, Mizuno N, Fujita T, et al. MSC/ECM cellular complexes induce periodontal tissue regeneration. J Dent Res. 2017:96(9):984-91.

7. Motoike S, Kajiya M, Komatsu N, Takewaki M, Horikoshi S, Matsuda S, Ouhara K, Iwata T, Takeda K, Fujita T, et al. Cryopreserved clumps of mesenchymal stem cell/extracellular matrix complexes retain osteogenic capacity and induce bone regeneration. Stem Cell Res Ther. 2018;9(1):73.

8. Baker BM, Chen CS. Deconstructing the third dimension: how 3D culture microenvironments alter cellular cues. J Cell Sci. 2012;125(Pt 13):3015-24.

9. Lv H, Li L, Sun M, Zhang Y, Chen L, Rong Y, Li Y. Mechanism of regulation of stem cell differentiation by matrix stiffness. Stem Cell Res Ther. 2015;6:103.

10. Engler AJ, Sen S, Sweeney HL, Discher DE. Matrix elasticity directs stem cell lineage specification. Cell. 2006;126:677-89.

11. McBeath R, Pirone DM, Nelson CM, Bhadriraju K, Chen CS. Cell shape, cytoskeletal tension, and RhoA regulate stem cell lineage commitment. Dev Cell. 2004:6(4):483-95.

12. Gao L, McBeath R, Chen CS. Stem cell shape regulates a chondrogenic versus myogenic fate through Rac1 and N-cadherin. Stem Cells. 2010;28(3):564-72.

13. Dong J, Feldmann G, Huang J, Wu S, Zhang N, Comerford SA, Gayyed MF, Anders RA, Maitra A, Pan D. Elucidation of a universal size-control mechanism in Drosophila and mammals. Cell. 2007;130:1120-33.

14. Yu FX, Zhao B, Guan KL. Hippo pathway in organ size control, tissue homeostasis, and cancer. Cell. 2015:163:811-28.

15. Zhao B, Wei X, Li W, Udan RS, Yang Q, Kim J, Xie J, Ikenoue T, Yu J, Li L, et al. Inactivation of YAP oncoprotein by the hippo pathway is involved in cell contact inhibition and tissue growth control. Genes Dev. 2007;21 (21):274761.

16. Halder G, Dupont S, Piccolo S. Transduction of mechanical and cytoskeletal cues by YAP and TAZ. Nat Rev Mol Cell Biol. 2012;13(9):591-600.

17. Panciera T, Azzolin L, Cordenonsi M, Piccolo S. Mechanobiology of YAP and TAZ in physiology and disease. Nat Rev Mol Cell Biol. 2017;18(12):758-70.

18. Wada K-I, Itoga K, Okano T, Yonemura S, Sasaki H. Hippo pathway regulation by cell morphology and stress fibers. Development. 2011;138:3907-14.

19. Aragona M, Panciera T, Manfrin A, Giulitti S, Michielin F, Elvassore N, Dupont S, Piccolo S. A mechanical checkpoint controls multicellular growth through YAP/TAZ regulation by actin-processing factors. Cell. 2013;154(5):1047-59.

20. Wang L, Luo JY, Li B, Tian XY, Chen L, Huang Y, Liu J, Deng D, Lau CW, Wan $\mathrm{S}$, et al. Integrin-YAP/TAZ-JNK cascade mediates atheroprotective effect of unidirectional shear flow. Nature. 2016;540:579-82. 
21. Dupont S, Morsut L, Aragona M, Enzo E, Giulitti S, Cordenonsi M, Zanconato F, Le Digabel J, Forcato M, Bicciato S, et al. Role of YAP/TAZ in mechanotransduction. Nature. 2011;474:179-83.

22. Tang Y, Rowe RG, Botvinick EL, Kurup A, Putnam AJ, Seiki M, Weaver VM Keller ET, Goldstein S, Dai J, et al. MT1-MMP-dependent control of skeletal stem cell commitment via a beta1-integrin/YAP/TAZ signaling axis. Dev Cell. 2013;25(4):402-16

23. Zhao B, Li L, Wang L, Wang CY, Yu J, Guan KL. Cell detachment activates the hippo pathway via cytoskeleton reorganization to induce anoikis. Genes Dev. 2012;26(1):54-68.

24. Kajiya M, Komatsuzawa H, Papantonakis A, Seki M, Makihira S, Ouhara K, Kusumoto Y, Murakami S, Taubman MA, Kawai T. Aggregatibacter actinomycetemcomitans Omp29 is associated with bacterial entry to gingival epithelial cells by F-actin rearrangement. PLoS One. 2011;6(4):e18287.

25. Hong JH, Hwang ES, McManus MT, Amsterdam A, Tian Y, Kalmukova R, Mueller E, Benjamin T, Spiegelman BM, Sharp PA, et al. TAZ, a transcriptional modulator of mesenchymal stem cell differentiation. Science. 2005; 309(5737):1074-8.

26. Sun Z, Guo SS, Fassler R. Integrin-mediated mechanotransduction. J Cell Biol. 2016;215(4):445-56.

27. Lee CH, Shah B, Moioli EK, Mao JJ. CTGF directs fibroblast differentiation from human mesenchymal stem/stromal cells and defines connective tissue healing in a rodent injury model. J Clin Invest. 2010;120(9):3340-9.

28. Cesarz Z, Tamama K. Spheroid culture of mesenchymal stem cells. Stem Cells Int. 2016:2016:9176357.

29. Sart S, Tsai AC, Li Y, Ma T. Three-dimensional aggregates of mesenchymal stem cells: cellular mechanisms, biological properties, and applications. Tissue Eng Part B Rev. 2014;20(5):365-80.

30. Murphy KC, Whitehead J, Falahee PC, Zhou D, Simon SI, Leach JK. Multifactorial experimental design to optimize the anti-inflammatory and proangiogenic potential of mesenchymal stem cell spheroids. Stem Cells. 2017;35(6):1493-504

31. Nzou G, Wicks RT, Wicks EE, Seale SA, Sane CH, Chen A, Murphy SV, Jackson JD, Atala AJ. Human cortex spheroid with a functional blood brain barrier for high-throughput neurotoxicity screening and disease modeling. Sci Rep. 2018;8(1):7413

32. Shinozawa T, Yoshikawa HY, Takebe T. Reverse engineering liver buds through self-driven condensation and organization towards medical application. Dev Biol. 2016;420(2):221-9.

33. Murphy KC, Hoch Al, Harvestine JN, Zhou D, Leach JK. Mesenchymal stem cell spheroids retain osteogenic phenotype through alpha2beta1 signaling. Stem Cells Transl Med. 2016;5(9):1229-37.

34. Takebe T, Enomura M, Yoshizawa E, Kimura M, Koike H, Ueno Y, Matsuzaki T, Yamazaki T, Toyohara T, Osafune K, et al. Vascularized and complex organ buds from diverse tissues via mesenchymal cell-driven condensation. Cell Stem Cell. 2015;16(5):556-65.

35. Boeuf S, Richter W. Chondrogenesis of mesenchymal stem cells: role of tissue source and inducing factors. Stem Cell Res Ther. 2010;1(4):31.

36. Karystinou A, Roelofs AJ, Neve A, Cantatore FP, Wackerhage H, De Bari C. Yes-associated protein (YAP) is a negative regulator of chondrogenesis in mesenchymal stem cells. Arthritis Res Ther. 2015;17:147.

37. Langenbach F, Handschel J. Effects of dexamethasone, ascorbic acid and beta-glycerophosphate on the osteogenic differentiation of stem cells in vitro. Stem Cell Res Ther. 2013;4(5):117.

38. Tang Y, Feinberg T, Keller ET, Li XY, Weiss SJ. Snail/slug binding interactions with YAP/TAZ control skeletal stem cell self-renewal and differentiation. Nat Cell Biol. 2016;18(9):917-29.

39. Hamidouche Z, Hay E, Vaudin P, Charbord P, Schule R, Marie PJ, Fromigue O. FHL2 mediates dexamethasone-induced mesenchymal cell differentiation into osteoblasts by activating Wnt/beta-catenin signaling-dependent Runx2 expression. FASEB J. 2008;22(11):3813-22.

40. Hamidouche Z, Fromigue O, Nuber U, Vaudin P, Pages JC, Ebert R, Jakob F, Miraoui H, Marie PJ. Autocrine fibroblast growth factor 18 mediates dexamethasone-induced osteogenic differentiation of murine mesenchymal stem cells. J Cell Physiol. 2010;224(2):509-15

41. Hamidouche Z, Fromigue O, Ringe J, Haupl T, Marie PJ. Crosstalks between integrin alpha 5 and IGF2/IGFBP2 signalling trigger human bone marrowderived mesenchymal stromal osteogenic differentiation. BMC Cell Biol. 2010;11:44.

42. Hamidouche $Z$, Fromigue $O$, Ringe J, Haupl T, Vaudin P, Pages JC, Srouji S, Livne E, Marie PJ. Priming integrin alpha5 promotes human mesenchymal stromal cell osteoblast differentiation and osteogenesis. Proc Natl Acad Sci U S A. 2009;106(44):18587-91.

43. Davidson JM, LuValle PA, Zoia O, Quaglino D Jr, Giro M. Ascorbate differentially regulates elastin and collagen biosynthesis in vascular smooth muscle cells and skin fibroblasts by pretranslational mechanisms. J Biol Chem. 1997;272(1):345-52.

44. Franceschi RT, lyer BS. Relationship between collagen synthesis and expression of the osteoblast phenotype in MC3T3-E1 cells. J Bone Miner Res. 1992;7(2):235-46.

45. Calvo F, Ege N, Grande-Garcia A, Hooper S, Jenkins RP, Chaudhry SI, Harrington K, Williamson P, Moeendarbary E, Charras G, et al. Mechanotransduction and YAP-dependent matrix remodelling is required for the generation and maintenance of cancer-associated fibroblasts. Nat Cell Biol. 2013;15:637-46.

46. Liu F, Lagares D, Choi KM, Stopfer L, Marinkovic A, Vrbanac V, Probst CK, Hiemer SE, Sisson TH, Horowitz JC, et al. Mechanosignaling through YAP and TAZ drives fibroblast activation and fibrosis. Am J Physiol Lung Cell Mol Physiol. 2015;308(4):L344-57.

47. Mannaerts I, Leite SB, Verhulst S, Claerhout S, Eysackers N, Thoen LF, Hoorens A, Reynaert H, Halder G, van Grunsven LA. The hippo pathway effector YAP controls mouse hepatic stellate cell activation. J Hepatol. 2015; 63(3):679-88.

48. Bertero T, Cottrill KA, Lu Y, Haeger CM, Dieffenbach P, Annis S, Hale A, Bhat $B$, Kaimal $V$, Zhang YY, et al. Matrix remodeling promotes pulmonary hypertension through feedback mechanoactivation of the YAP/TAZ-miR130/301 circuit. Cell Rep. 2015;13(5):1016-32

49. Bertero T, Oldham WM, Cottrill KA, Pisano S, Vanderpool RR, Yu Q, Zhao J, Tai Y, Tang Y, Zhang YY, et al. Vascular stiffness mechanoactivates YAP/TAZdependent glutaminolysis to drive pulmonary hypertension. J Clin Invest. 2016;126(9):3313-35.

50. Jorgenson AJ, Choi KM, Sicard D, Smith KM, Hiemer SE, Varelas X, Tschumperlin DJ. TAZ activation drives fibroblast spheroid growth, expression of profibrotic paracrine signals, and context-dependent ECM gene expression. Am J Physiol Cell Physiol. 2017;312(3):C277-85.

51. Takarada T, Nakazato R, Tsuchikane A, Fujikawa K, lezaki T, Yoneda Y, Hinoi E. Genetic analysis of Runx2 function during intramembranous ossification. Development. 2016;143(2):211-8.

52. Leeming DJ, Henriksen K, Byrjalsen I, Qvist P, Madsen SH, Garnero P, Karsdal MA. Is bone quality associated with collagen age? Osteoporos Int. 2009; 20(9):1461-70
Ready to submit your research? Choose BMC and benefit from:

- fast, convenient online submission

- thorough peer review by experienced researchers in your field

- rapid publication on acceptance

- support for research data, including large and complex data types

- gold Open Access which fosters wider collaboration and increased citations

- maximum visibility for your research: over $100 \mathrm{M}$ website views per year

At BMC, research is always in progress.

Learn more biomedcentral.com/submissions 